\title{
Preventive analgesia for postoperative pain control: a broader concept
}

This article was published in the following Dove Press journal:

Local and Regional Anesthesia

29 April 2014

Number of times this article has been viewed

\author{
Nalini Vadivelu' \\ Sukanya Mitra ${ }^{2}$ \\ Erika Schermer ${ }^{3}$ \\ Vijay Kodumudi ${ }^{4}$ \\ Alan David Kaye ${ }^{5}$ \\ Richard D Urman ${ }^{6}$ \\ 'Department of Anesthesiology, \\ Yale University School of Medicine, \\ New Haven, CT, USA; ${ }^{2}$ Department \\ of Anesthesia and Intensive Care, \\ Government Medical College and \\ Hospital, Chandigarh, India; ${ }^{3}$ Yale \\ College, New Haven, ${ }^{4}$ School of \\ Liberal Arts and Science, University of \\ Connecticut, Storrs, CT, ${ }^{5}$ Department \\ of Anesthesiology, Louisiana State \\ University School of Medicine, \\ New Orleans, LA, ${ }^{6}$ Department of \\ Anesthesiology, Perioperative and \\ Pain Medicine, Brigham and Women's \\ Hospital, Boston, MA, USA
}

\begin{abstract}
Pain from surgical procedures occurs as a consequence of tissue trauma and may result in physical, cognitive, and emotional discomfort. Almost a century ago, researchers first described a possible relationship between intraoperative tissue damage and an intensification of acute pain and long-term postoperative pain, now referred to as central sensitization. Nociceptor activation is mediated by chemicals that are released in response to cellular or tissue damage. Pre-emptive analgesia is an important concept in understanding treatment strategies for postoperative analgesia. Pre-emptive analgesia focuses on postoperative pain control and the prevention of central sensitization and chronic neuropathic pain by providing analgesia administered preoperatively but not after surgical incision. Additional research in pre-emptive analgesia is warranted to better determine good outcome measurements and a better appreciation with regard to treatment optimization. Preventive analgesia reduces postoperative pain and consumption of analgesics, and this appears to be the most effective means of decreasing postoperative pain. Preventive analgesia, which includes multimodal preoperative and postoperative analgesic therapies, results in decreased postoperative pain and less postoperative consumption of analgesics.
\end{abstract}

Keywords: preventive analgesia, central sensitization, chronic pain, pre-emptive analgesia, multimodal analgesia, acute pain

\section{Introduction}

There has been much confusion and controversy regarding the terms pre-emptive analgesia and preventive analgesia. While the aim of both is to decrease postoperative pain while decreasing the consumption of analgesics after surgery and reduction of long-term pain sensitization, this review attempts to clarify and differentiate these two concepts. Recent understandings in pre-emptive analgesia have defined it as an intervention given before incision or surgery, given that it is more effective than the same treatment administered after incision or surgery. ${ }^{1,2}$ It is important to remember the timing of pre-emptive analgesia in that it is an antinociceptive treatment given prior to incision or surgery. This helps to prevent the development of altered processing of afferent input, which would otherwise amplify postoperative pain. ${ }^{3}$ Almost a century ago, Crile ${ }^{4}$ first described a possible relationship between intraoperative tissue damage and an intensification of acute pain and long-term postoperative pain, which is now referred to as central sensitization. He advocated a multimodal approach, including drugs and use of regional blocks with local anesthetics, in addition to general anesthesia. His rationale was to prevent intraoperative nociception with what we now call preemptive analgesia; lack of such intervention can lead to changes in the central nervous
Correspondence: Richard D Urman Harvard Medical School, Brigham and Women's Hospital, 75 Francis Street, Boston, MA, 021I5, USA

Tel +l 6177328222

Email rurman@partners.org 
system during surgery and formation of painful scars. Wall first proposed the concept of pre-emptive preoperative analgesia in 1988..$^{5}$ Pre-emptive analgesia given before incision and surgery focuses on managing perioperative pain, as well as conferring neuroprotective properties. ${ }^{6}$

The concept of pre-emptive analgesia is based on advances and research in the basic science of pain and evidence-based clinical research. It has now been refined and evolved to a broader concept that surgical incision alone is not the trigger for central sensitization. ${ }^{7}$ Other factors, such as preoperative pain and additional painful noxious intraoperative inputs such as retraction, as well as postoperative inflammatory processes, related peripheral and central neuromodulators, and ectopic neural activity ${ }^{8}$ can all cause an intensification of acute pain and long-term postoperative pain as a result of central sensitization. At present, there are several multimodal strategies covering the perioperative period that can help to decrease postoperative pain and minimize consumption of analgesics. Preventive analgesia is now considered a more appropriate term because it encompasses all perioperative efforts to decrease postoperative pain and decrease consumption of analgesics. Preventive analgesia could be provided by a number of means, including no treatment at all, placebo treatment, or multimodal treatment that can be given to decrease postoperative pain and reduce analgesic consumption. This treatment can be given at any time in the perioperative period, including the preoperative period, which results in a duration of action greater than that expected for the particular intervention given to decrease postoperative pain and to reduce consumption of analgesics. ${ }^{1,2}$ The molecular basis of central sensitization is an important concept for the understanding of pre-emptive analgesia and preventive analgesia. Central sensitization provides the neurobiological basis for much of the pain suffered by patients long after normal tissue healing has occurred. ${ }^{9}$

Left untreated, acute pain can lead to long-term emotional and psychological distress and has the potential to develop into a chronic pain state which is much more difficult to manage. ${ }^{10}$ Central sensitization can occur for a variety of reasons, ranging from preoperative pain to intraoperative tissue injury and postoperative inflammatory processes that can occur immediately after surgery up to several weeks later. ${ }^{11}$

\section{Molecular mechanisms of central sensitization}

In 1986, Woolf and Wall demonstrated that the motor and sensory changes found after peripheral tissue injury were linked to peripheral activation of $\mathrm{C}$ afferents. ${ }^{12}$ Tissue injury results in initiation of nociceptive signals to the dorsal horn of the spinal cord by A fiber nociceptors and by polymodal $\mathrm{C}$ fiber nociceptors. ${ }^{13}$ Activation of nociceptors is mediated by chemicals that are released in response to cellular or tissue damage. For example, injured cells release leukotrienes and potassium, platelets release serotonin, and vascular injury causes a release of bradykinin from plasma. ${ }^{14}$ These nociceptive signals release neurotransmitters and substances which amplify the responses from the dorsal horn neurons, with resultant further input into the dorsal horn leading to central sensitization. These substances include substance P, calcitonin gene-related peptide, and the excitatory amino acids, glutamate and aspartate. ${ }^{15-17}$ Central sensitization, after tissue injury in both neuropathic and inflammatory pain, is a cause of increased pain sensitivity described as hyperalgesia and tactile allodynia, where there is pain to light touch and persistent chronic pain. In addition to increased excitability, there is a reduction in inhibitory transmission from decreases in inhibitory interneurons. ${ }^{18}$ Given that clinicians have determined a role for central sensitization in long-term changes of the central nervous system, treatment strategies involving modulation of central sensitization are important both in the short term and the long term. However, in some disorders, there is no evidence of nerve lesions or tissue injury as a trigger for pain hypersensitivity. For example, in disorders such as fibromyalgia or irritable bowel syndrome, ${ }^{19}$ it appears that the pain is driven by central amplification of peripheral autonomous inputs caused by central sensitization occurring autonomously.

\section{Preoperative strategy for management of postoperative pain}

For effective postoperative pain control, a physician must include a prospective and predesigned strategy for management of pain and discuss the plan with the patient. This plan would include discussion of postoperative and perioperative management as well as physical therapy. ${ }^{6}$ Before the operation, the clinician should also take a thorough patient history, including factors such as prior responses to pain, past contact with analgesics, current medications, fears or concerns regarding future pain, and any additional pertinent information. Such an assessment helps to pinpoint factors that may influence the severity of pain felt and the patient's response to it, as well as allow for open communication and alleviation of fear prior to surgery. ${ }^{14}$

Pain is more than a simple bodily reaction to noxious stimuli; rather, it is an intricate and individualized experience. Patients undergoing a surgical procedure are predisposed to 
pain postoperatively for a number of reasons. For example, pre-existing pain (both acute and chronic), psychological influences, fear of recurring additional pain, neurovascular tissue damage from a prior operation, as well as the extent of the surgery can all contribute to major postoperative discomfort. $^{3}$

\section{Adverse clinical effects of postsurgical pain}

Pain from surgical procedures occurs as a consequence of tissue trauma and may further result in physical, cognitive, and emotional discomfort. ${ }^{20}$ The outcome of a surgical procedure can be affected as a result of changes arising from pain. These pain-related changes can affect insulin, cortisol, catecholamine, and other hormone levels. ${ }^{21}$ Decrease in physical mobility can predispose an individual to pneumonia, ${ }^{22}$ and muscle splinting has the potential to decrease blood flow to an extremity, resulting in thrombosis or embolism. ${ }^{23}$ Muscles can be further damaged by spasm, atrophy, and impairment of metabolism. ${ }^{21}$ Muscles controlling urinary bladder motility can become impaired, resulting in urinary retention. Coronary vasoconstriction from activation of the sympathetic nervous system can cause cardiovascular effects such as angina or ischemia. Furthermore, unresolved postoperative pain may give rise to sleep deprivation and psychological issues such as anxiety and depression. ${ }^{24}$

\section{Chronic postsurgical pain}

Chronic postsurgical pain (CPSP) generally refers to pain that persists for 3-6 months after surgery. It affects a large number of individuals and disrupts their quality of life. More research is warranted to better understand the mechanisms of chronic postsurgical pain. CPSP can occur due to inflammatory processes or initiation of neuropathic pain from peripheral nerve damage during surgery, and both causing intense tissue injury. CPSP occurs in $10 \%-50 \%$ of patients after surgery. ${ }^{25}$ Several risk factors have been associated with the development of CPSP, and include psychosocial factors, sex, age, genetic predisposition, and level of pre-existing pain. It is well established that multimodal analgesia and use of surgical techniques that avoid nerve damage are beneficial for preventing long-term postsurgical pain.

\section{Prevention of postsurgical pain by preventive analgesia}

Woolf and Chong ${ }^{26}$ and Wall ${ }^{5}$ hypothesized that an antinociceptive intervention given pre-emptively, ie, before the start of surgery, would decrease the intensity of postoperative pain, decrease hyperalgesia, and prevent central sensitization when compared with the same intervention given after the start of surgery. However, subsequent clinical studies of the hypothesis of pre-emptive analgesia by comparing antinociceptive interventions given before incision versus after incision yielded contradictory results. Preventive analgesia encompassing multimodal antinociceptive interventions, started preoperatively and given for an increased duration including the postoperative period, was found to be more effective in terms of decreasing postoperative pain and reducing analgesic consumption in the postoperative period. Preventive analgesia employing multimodal pain management for a longer duration and combining multiple analgesic treatments reduces untoward side effects, allowing more rapid recovery and earlier discharge from hospital. ${ }^{27}$

\section{Substances and interventions used for preventive analgesia: clinical studies}

Central sensitization and hyperalgesia induced by the incision can be attenuated by several agents in the perioperative period, including N-methyl-D-aspartic acid (NMDA) receptor antagonists such as dextromethorphan and ketamine. These drugs have been shown to have preventive analgesic effects, such as decreasing both acute and chronic postoperative pain. Other substances and interventions include epidural analgesia combined with systemic ketamine. Lavand'homme et $\mathrm{al}^{28}$ were the first to demonstrate that postoperative systemic ketamine along with intraoperative epidural analgesia provides preventive analgesia after major gastrointestinal surgery and reduces the risk of development of chronic (persistent) postsurgical pain.

Another interesting preventive analgesia intervention was multimodal treatment in patients undergoing thoracotomy, in whom epidural administration of neostigmine was started preoperatively and continued postoperatively in combination with morphine or bupivacaine, which reduced analgesic consumption significantly. ${ }^{29}$ Although further studies are necessary to demonstrate clinical significance, there have been reports of systemic gabapentin attenuating hyperalgesia after surgery, decreasing consumption of opioids, and reducing pain scores. ${ }^{30,31}$

Preventive analgesia was also demonstrated by Koppert et al, who showed that systemic lidocaine given perioperatively decreased postoperative pain scores and reduced overall morphine consumption in the first 72 hours after surgery. ${ }^{32}$ It appeared that peripheral sensitization was suppressed with this treatment. It must be emphasized that 
some treatments may reduce the intensification of chronic postsurgical pain but not that of acute postoperative pain. It has been shown that modulators of central sensitization, such as spinal clonidine and ketamine, can reduce the incidence of persistent postoperative pain, but do not seem to significantly reduce acute postsurgical pain. ${ }^{33}$

\section{Studies in pre-emptive analgesia}

Surgery, which induces central sensitization, has an incisional and an inflammatory phase, the latter being a reaction to damaged tissue. ${ }^{3}$ Therefore, it has been suggested that antinociceptive protection provided by pre-emptive treatment should extend well into the postoperative period to cover the inflammatory phase in order to be effective. Some animal studies had demonstrated that anesthetic techniques which profoundly reduce the amount of pain information getting into the spinal cord and brain can prevent central sensitization and reduce subsequent pain-related behavior when administered prior to the painful stimulus. ${ }^{34}$ However, other animal studies did not show the same results. In a rat model involving a brief surgical incision in the plantar hind paw, there was no difference between preincisional and postincisional treatment with intrathecal bupivacaine or intrathecal morphine. ${ }^{35}$

Several pre-emptive analgesic regimens have been tried in humans. These include intravenous doses of opioids, ${ }^{36,37}$ peripheral nerve blocks, ${ }^{38}$ local infiltration of the surgical site, ${ }^{39,40}$ epidural administration of opioids and local anesthetics, ${ }^{41-43}$ and multimodal combinations. ${ }^{44}$ Treatment with nonsteroidal anti-inflammatory drugs given systemically and NMDA receptor antagonists has also been tried preemptively. In humans, these trials, along with meta-analytic studies, demonstrate the need for perioperative and not just preoperative analgesic interventions. In a large meta-analysis, Ong et al ${ }^{45}$ combined 66 studies (total $n=3,261$ ) and stratified them according to type of analgesic intervention and outcome measures. The authors found the most robust analgesic effect for pre-emptive pain control was with epidural analgesia, followed by nonsteroidal anti-inflammatory drugs and local anesthetic wound infiltration. Pre-emptive opioids and systemic NMDA antagonists were not found to be efficacious.

There are additional meta-analytic studies of perioperative analgesic interventions to provide preventive analgesia. Møiniche et $\mathrm{al}^{1}$ reviewed 80 randomized controlled trials and concluded that there was no superiority of pre-emptive analgesia given before incision when compared with analgesic treatment given after incision. In addition, Dahl et al compared pain scores and analgesic requirements, and reported no significant decrease in pain scores or analgesic requirements if analgesic intervention was given preoperatively versus postoperatively.

The reason for the differences in the results of the metaanalysis by Ong et al and that of Dahl et al and Møiniche et al lie either in the different approaches used to calculate pain scores or in the different patient inclusion criteria used. . $^{1,45,48}$

\section{Discussion}

A large body of literature exists comparing preincisional and postincisional analgesic interventions. The majority of the literature shows discrepancies in the results of both animal and human studies regarding the beneficial effects of pre-emptive analgesia. The overarching theory that likely explains the limits of the effectiveness of pre-emptive analgesia is that sensitization from incisional pain causes a shorter duration of peripheral sensitization and distinct central sensitization by initial excitation of primary afferent fibers. Surgical incision, although still a type of tissue injury, may not be as severe as the intense tissue injury seen in nerve injury and inflammation, the latter two being more persistent, of longer duration, and more challenging to treat. It appears that preventive analgesia involving perioperative pain control with increased duration of analgesic treatment employing multimodal pain control techniques is more effective than sole administration of pre-emptive analgesia before the incision is made. Preventive analgesia helps to decrease hyperalgesia and peripheral and central sensitization. The duration and efficacy of a perioperative analgesic regimen is more important than the preoperative timing of an analgesic intervention alone. Preventive analgesia, a broader definition and a more inclusive term, encompasses any analgesic regimen in the perioperative period given to control sensitization induced by pain. ${ }^{46}$ Clinical practice points and a future research agenda are outlined in Table 1.

The most effective means by which to significantly decrease pain is a combination of pre-emptive analgesia and multimodal pain management. Multimodal therapy allows for lower dosages of any one medication to be used in combination, which reduces the risk of a significant side effect arising from administration of a single analgesic drug. Preventive analgesia includes pre-emptive analgesia to reduce sensitization caused by pain. Effective multimodal analgesia is an important component of preventive analgesia, resulting in an increased duration of action of analgesic drugs to decrease long-term pain sensitivity at the peripheral and central levels. 
Table I Clinical practice points and research agenda

\section{Clinical practice points}

- Timing of administration of pre-emptive analgesia is before the incision or surgery. Pre-emptive analgesia provides improved analgesia postoperatively compared with the identical analgesic treatment after incision or surgery.

- Multimodal analgesia allows for lower doses of any one medication to be used in combination, thus decreasing side effects.

- Preventive analgesia can be provided by an intervention given before or after incision and surgery, whether it be a placebo, no treatment, or analgesic treatment that reduces analgesic use or postoperative pain for a period longer than the duration of action for the intervention.

- Duration of treatment and effective analgesic regimens are the two important considerations in the administration of preventive analgesia.

\section{Research agenda}

- There is an urgent need for preventive analgesic research evaluating which analgesic regimens would decrease the phenomenon of hyperalgesia and pain after surgery most effectively, and the multimodal therapies that would decrease or prevent long-term pain after surgery.

- More research is required to improve postoperative pain control by preventive analgesia with the use of another treatment, such as peripheral nerve blocks focusing on duration of postoperative analgesia.

Preventive analgesia is not time-constrained and involves the use of analgesic interventions perioperatively. Without a proper pain management plan, postoperative pain has the potential to result in chronic pain, with long-term negative consequences for the patient. ${ }^{39,47}$ Prevention of this pain has been dubbed as the "holy grail of anesthesiology", ${ }^{10}$ with more studies currently underway. "Preventive analgesia" may be a more appropriate term for all these efforts covering the perioperative period rather than the previously used term "pre-emptive analgesia" which has narrower connotations. ${ }^{48,49}$

\section{Disclosure}

The authors report no conflicts of interest in this work.

\section{References}

1. Møiniche S, Kehlet H, Dahl JB. A qualitative and quantitative systematic review of pre-emptive analgesia for postoperative pain relief: the role of timing of analgesia. Anesthesiology. 2002;96:725-741.

2. Katz J, McCartney CJ. Current status of pre-emptive analgesia. Curr Opin Anaesthesiol. 2002;15:435-441.

3. Kissin I. Pre-emptive analgesia. Anesthesiology. 2000;93:1138-1143.

4. Crile GW. In: Austin A. Man - An Adaptive Mechanism. New York, NY, USA: The Macmillan Company; 1916.

5. Wall PD. The prevention of postoperative pain. Pain. 1988;33: 289-290.

6. Gottschalk A. Update on pre-emptive analgesia. Tech Reg Anesth Pain Manag. 2003;7:116-121.

7. Katz J. Pre-emptive analgesia: evidence, current status and future directions. Eur J Anaesthesiol Suppl. 1995;10:8-13.
8. Katz J, Seltzer Z. Transition from acute to chronic postsurgical pain: risk factors and protective factors. Expert Rev Neurother. 2009;9: 723-744.

9. Woolf CJ. Central sensitization: implications for the diagnosis and treatment of pain. Pain. 2011;152(Suppl 3):S2-S15.

10. Cohen P, Raja SN. Prevention of chronic postsurgical pain. The ongoing search for the holy grail of anesthesiology. Anesthesiology. 2013;118:241-243.

11. Katz J, Clarke H, Seltzer Z. Review article. Preventive analgesia: quo vadimus? Anesth Analg. 2011;113:1242-1253.

12. Woolf CJ, Wall PD. Relative effectiveness of $\mathrm{C}$ primary afferent fibers of different origins in evoking a prolonged facilitation of the flexor reflex in the rat. $J$ Neurosci. 1986;6:1433-1442.

13. Dahl JB, Møiniche S. Pre-emptive analgesia. Br Med Bull. 2004;71: $13-27$.

14. Woolf CJ. Evidence for a central component of post-injury pain hypersensitivity. Nature. 1983;306:686-688.

15. Costigan M, Woolf CJ. Pain: molecular mechanisms. J Pain. 2000; 1(Suppl 3):35-44.

16. Woolf CJ, Salter MW. Neuronal plasticity: increasing the gain in pain. Science. 2000;288:1765-1769.

17. Julius D, Basbaum AI. Molecular mechanisms of nociception. Nature. 2001;413:203-210.

18. Simonnet G. Pre-emptive antihyperalgesia to improve pre-emptive analgesia. Anesthesiology. 2008;108:354-356.

19. Woolf CJ. Central sensitization. Anesthesiology. 2007;106:864-867.

20. Parsons B, Schaefer C, Mann R, et al. Economic and humanistic burden of post-trauma and post-surgical neuropathic pain among adults in the United States. J Pain Res. 2013;6:459-469.

21. Dunwoody CJ, Krenzischek DA, Pasero C, Rathmell JP, Polomano RC. Assessment, physiological monitoring, and consequences of inadequately treated acute pain. Pain Manag Nurs. 2008;9:S11-S21.

22. Pessaux $P$, van den Broek MA, Wu T, et al. Identification and validation of risk factors for postoperative infectious complications following hepatectomy. J Gastrointest Surg. 2013;17:1907-1916.

23. Bader P, Echtle D, Fonteyne V, et al. Post-Operative Pain Management In: Guidelines on Pain Management. Arnhem, The Netherlands: European Association of Urology; 2010.

24. Akkaya T, Ozkan D. Chronic post-surgical pain. Agri. 2009;21:1-9.

25. Kehlet H, Jensen TS, Woolf CJ. Persistent postsurgical pain: risk factors and prevention. Lancet. 2006;367:1618-1625.

26. Woolf CJ, Chong MS. Pre-emptive analgesia - treating postoperative pain by preventing the establishment of central sensitization. Anesth Analg. 1993;77:362-379.

27. Buvanendran A, Kroin JS. Multimodal analgesia for controlling acute postoperative pain. Curr Opin Anaesthesiol. 2009;22:588-593.

28. Lavand'homme P, De Kock M, Waterloos H. Intraoperative epidural analgesia combined with ketamine provides effective preventive analgesia in patients undergoing major digestive surgery. Anesthesiology. 2005;103:813-820.

29. Chia YY, Chang TH, Lui K, Chang HC, Ko NH, Wang YM. The efficacy of thoracic epidural neostigmine infusion after thoracotomy. Anesth Analg. 2006;102:201.

30. Seib RK, Paul JE. Preoperative gabapentin for postoperative analgesia: a meta-analysis. Can J Anaesth. 2006;53:461-469.

31. Rowbotham DJ. Editorial II: Gabapentin: a new drug for postoperative pain? Br J Anaesth. 2006;96:152-155.

32. Koppert W, Weigand M, Neumann F, et al. Perioperative intravenous lidocaine has preventive effects on postoperative pain and morphine consumption after major abdominal surgery. Anesth Analg. 2004;98: $1050-1055$.

33. Lavand'homme P. Perioperative pain. Curr Opin Anaesthesiol. 2006;19: 556-561.

34. Dickenson AH, Le Bars D. Supraspinal morphine and descending inhibitions acting on the dorsal horn of the rat. J Physiol. 1987;384:81-107.

35. Brennan T, Umali E, Zahn P. Comparison of pre- versus post-incision administration of intrathecal bupivacaine and intrathecal morphine in a rat model of postoperative pain. Anesthesiology. 1997;87:1517-1528. 
36. Richmond CE, Bromley LM, Woolf CJ. Preoperative morphine preempts postoperative pain. Lancet. 1993;342:73-75.

37. Fu ES, Miguel R, Scharf JE. Pre-emptive ketamine decreases postoperative narcotic requirements in patients undergoing abdominal surgery. Anesth Analg. 1997;84:1086-1090.

38. Bugedo GJ, Carcamo CR, Mertens RA, Dagnino JA, Munoz HR. Preoperative percutaneous ilioinguinal and iliohypogastric nerve block with $0.5 \%$ bupivacaine for post-herniorrhaphy pain management in adults. Reg Anesth. 1990;15:130-133.

39. Ejlersen E, Andersen HB, Eliasen K, Mogensen T. A comparison between preincisional and postincisional lidocaine infiltration and postoperative pain. Anesth Analg. 1992;74:495-498.

40. Tverskoy M, Cozacov C, Ayache M, Bradley EL, Kissin I. Postoperative pain after inguinal herniorrhaphy with different types of anesthesia. Anesth Analg. 1990;70:29-35.

41. Jahangiri M, Jayatuna AP, Bradley JW, Dark CH. Prevention of phantom pain after major lower limb amputation by epidural infusion of diamorphine, clonidine, and bupivacaine. Ann R Coll Surg Engl. 1994; 76:324-326.

42. Gottschalk A, Smith DS, Jobes DR, et al. Pre-emptive epidural analgesia and recovery from radical prostatectomy: a randomized controlled trial. JAMA. 1998;279:1076-1082.
43. Kundra P, Gurnani A, Bhattacharya A. Pre-emptive epidural morphine for postoperative pain relief after lumbar laminectomy. Anesth Analg. 1997;85:135-138

44. Kehlet H, Dahl JB. The value of "multimodal" or "balanced analgesia" in postoperative pain treatment. Anesth Analg. 1993;77: 1048-1056.

45. Ong CK, Lirk P, Seymour RA, Jenkins BJ. The efficacy of preemptive analgesia for acute postoperative pain management: a meta-analysis. Anesth Analg. 2005;100:757-773.

46. Pogatzki-Zahn EM, Zahn PK. From pre-emptive to preventive analgesia. Curr Opin Anaesthesiol. 2006;19:551-555.

47. Woolf CJ. Recent advances in the pathophysiology of acute pain. $B r J$ Anaesth. 1989;63:139-146.

48. Dahl JB, Kehlet H. Preventive analgesia. Curr Opin Anaesthesiol. 2011;24:331-338.

49. Mitra S, Ahuja V, Vadivelu N. Mechanisms of pain. In: Urman R, Vadivelu N, editors. Perioperative Pain Management. New York, NY, USA: Oxford University Press; 2013.
Local and Regional Anesthesia

\section{Publish your work in this journal}

Local and Regional Anesthesia is an international, peer-reviewed, open access journal publishing on the development, pharmacology, delivery and targeting and clinical use of local and regional anesthetics and analgesics. The journal welcomes submitted papers covering original research, basic science, clinical studies, reviews \& evaluations,

\section{Dovepress}

guidelines, expert opinion and commentary, case reports and extended reports. The manuscript management system is completely online and includes a very quick and fair peer-review system, which is all easy to use. Visit http://www.dovepress.com/testimonials.php to read real quotes from published authors. 